\title{
Metabolic Engineering of Rational Screened Saccharopolyspora spinosa for the Enhancement of Spinosyns A and D Production
}

\author{
Amit Kumar Jha, Anaya Raj Pokhrel, Amit Kumar Chaudhary, Seong-Whan Park ${ }^{1}$, Wan Je Cho ${ }^{1}$, and \\ Jae Kyung Sohng*
}

\begin{abstract}
Spinosyns $A$ and $D$ are potent ingredient for insect control with exceptional safety to non-target organisms. It consists of a 21-carbon tetracyclic lactone with forosamine and tri-Omethylated rhamnose which are derived from S-adenosylmethionine. Although previous studies have revealed the involvement of metK1 (S-adenosylmethionine synthetase), rmbA (glucose-1-phosphate thymidylyltransferase), and $r m b B$ (TDP-D-glucose-4, 6-dehydratase) in the biosynthesis of spinosad, expression of these genes into rational screened Saccharopolyspora spinosa (S. spinosa MUV) has not been elucidated till date. In the present study, S. spinosa MUV was developed to utilize for metabolic engineering. The yield of spinosyns $A$ and $D$ in S. spinosa MUV was $244 \mathrm{mg} \mathrm{L}^{-1}$ and $129 \mathrm{mg} \mathrm{L}^{-1}$, which was 4.88-fold and 4.77-fold higher than that in the wild-type ( $50 \mathrm{mg} \mathrm{L}^{-1}$ and $\left.27 \mathrm{mg} \mathrm{L}^{-1}\right)$, respectively. To achieve the better production; positive regulator metK1-sp, $r m b A$ and $r m b B$ genes from Streptomyces peucetius, were expressed and co-expressed in S. spinosa MUV under the control of strong ermE* promoter, using an integration vector pSET152 and expression vector pIBR25, respectively. Herewith, the genetically engineered strain of $S$. spinosa MUV, produce spinosyns $A$ and $D$ up to $372 / 217 \mathrm{mg} \mathrm{L}^{-1}$ that is 7.44/8.03-fold greater than that of wild type. This result demonstrates the use of metabolic engineering on rationally developed high producing natural variants for the production.
\end{abstract}

\section{INTRODUCTION}

Spinosyn A and spinosyn D together known as spinosad; a bioinsecticide derived from the fermentation of the soil microorgan-

Institute of Biomolecule Reconstruction (iBR), Department of Pharmaceutical Engineering, Sun Moon University, Asan 333-708, Korea, ' ${ }^{1}$ ife Research Institute Dongbu Farm Hannong Co., Ltd., Daejeon 305-708, Korea

*Correspondence: sohng@sunmoon.ac.kr

Received 16 June, 2014; revised 19 August, 2014; accepted 19 August, 2014; published online 26 September, 2014

Keywords: metabolic engineering, metK1-sp, rmbA, rmbB ism Saccharopolyspora spinosa, discovered by Lilly in 1997 and is now widely used as a field pest control agent on many crops (Jin et al., 2009). Structurally, Spinosyns are macrolides consisting of a 21-carbon, tetracyclic lactone, to which two deoxysugars: tri-O-methylated rhamnose and forosamine are attached (Kirst et al., 1992). The most active and major components of the spinosyn family are spinosyns $A$ and $D$ which differs from each other by a single methyl substituent at position 6 of the polyketide; other factors in spinosyn family have different levels of methylation and are significantly less active (Madduri et al., 2001). Spinosad are highly effective against target insects and has an excellent environmental and mammalian toxicological profile (Crouse and Sparks, 1998; Sparks et al., 1998). Incorporation studies with ${ }^{13} \mathrm{C}$-labeled acetate, propionate, butyrate, and methionine established that spinosyns are assembled by a polyketide pathway and that the two $\mathrm{N}$-methyl groups of forosamine and the three $O$-methyl groups of tri-Omethylrhamnose are derived from S-adenosyl-methionine. Most of the genes involved in spinosad biosynthesis are clustered in a 74-kb region of the $S$. spinosa genome. The spinosad biosynthetic gene cluster contains five large genes encoding a type I polyketide synthase and 14 genes involved in sugar biosynthesis, sugar attachment to the polyketide, or cross-bridging of the polyketide. Four rhamnose biosynthetic genes, two of which are also necessary for forosamine biosynthesis, are located outside the spinosyn gene cluster (Waldron et al., 2001a; 2001b).

Emerging tools for synthetic biology and recombinant DNA technology has led to a number of strategies for metabolic engineering (Chaudhary et al., 2013; Koffas et al., 1999; Lee et al., 2013; Nielsen, 2001). The potential productivity of the actinomycetes is controlled by its genome and, therefore, the genome must be modified to increase the yield. Genome modification in Saccharopolyspora spinosa was achieved by duplication of gene cluster (18 kb) (Tang et al., 2011), genome shuffling (Jin et al., 2009) and overexpression of clustered genes (Luo et al., 2011; Xue et al., 2013) respectively. Heterologous expressions of the positive regulator S-adenosylmethionine synthetase (metK1) is found to be involved in enhancement of various secondary metabolites from different actinomycetes. (Jha et al., 2014; Kim et al., 2003; Lee et al., 2002; Okamoto et al., 2003; Wang et al., 2007; Zhao et al., 2006). The spnH, spnl, and spnK are the respective rhamnose 4'-, 2'-, and 3'-O-methyltransferase 
Table 1. Strains and plasmids used in this study

\begin{tabular}{|c|c|c|}
\hline Strain or plasmid & Description & Sources \\
\hline \multicolumn{3}{|l|}{ Bacterial strain } \\
\hline S. spinosa ATCC83543.1 & Wild type & ATCC \\
\hline S. spinosa MUV & Rational strain obtain by UV mutagenesis into S. spinosa 83543.1 & This study \\
\hline S. spinosa MUV IBR25 & Expression of plBR25 vector into S. spinosa MUV & This study \\
\hline S. spinosa MUV SIBR & pIBR25 with metK1-sp expressed into S. spinosa MUV & This study \\
\hline S. spinosa MUV RMBIBR & pIBR25 with $r m b A$ and $r m b B$ expressed into S. spinosa MUV & This study \\
\hline S. spinosa MUV SAM152 & pSET152 with metK1-sp integrated into S. spinosa MUV & This study \\
\hline S. spinosa MUV RMB152 & pSET152 with $r m b A$ and $r m b B$ integrated into S. spinosa MUV & This study \\
\hline \multicolumn{3}{|c|}{ 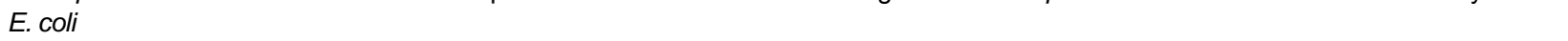 } \\
\hline XL1 Blue & General cloning host & Stratagene PBL company \\
\hline JM110 & Demethylation host & Stratagene Lajolla, USA \\
\hline \multicolumn{3}{|l|}{ Plasmid } \\
\hline pIBR25 & Streptomyces expression vector, with ermE* promoter and $T s r^{r}$ & SunMoon University \\
\hline pSIBR & pIBR25 based recombinant plasmid harboring metK1-sp & This study \\
\hline pRMBIBR & pIBR25 based recombinant plasmid harboring $r m b A$ and $r m b B$ & This study \\
\hline pSET152 & Streptomyces integration vector, with $\mathrm{ermE}^{\star}$ promoter and $\mathrm{Apr}^{r}$ & SunMoon University \\
\hline pSAM152 & pSET152 based recombinant plasmid harboring metK1-sp & This study \\
\hline pRMB152 & pSET152 based recombinant plasmid harboring $r m b A$ and $r m b B$ & This study \\
\hline
\end{tabular}

which are responsible for the final rhamnosyl methylation step in the biosynthesis of spinosyn (Huang et al., 2008; Kim et al., 2010). Moreover, glucose-1-phosphate thymidylyltransferase $(\mathrm{rmbA})$ and TDP-D-glucose-4,6-dehydratase $(\mathrm{rmbB})$ were also found to be overexpressed for altering the metabolic flux distribution in TDP-4-keto-6-deoxy-D-glucose, which is the common intermediate in TDP-deoxysugar biosynthetic pathways (Madduri et al., 2001; Pan et al., 2011). Similarly, UV irradiation is one of the strain improvement strategies through random mutation. UV radiation, in the range of $200-300 \mathrm{~nm}$, produces thymidine dimers and increases probability of deletion during the duplication process. UV is a very convenient and relatively safe mutagen (Kieser et al., 2000), however very few researchers have used this technique to overproduce spinosad (Jin et al., 2006; Liang et al., 2009). These examples imply that introduction of extra copies of biosynthetic gene clusters into a wild-type strain might be an effective approach to improve the yield of the corresponding product. Although these techniques have succeeded in generating many industrial strains, they never use natural variants, developed by UV irradiation for genetic manipulation.

In present study, the rational screened Saccharopolyspora spinosa MUV, a high producing strain was developed by UV mutagenesis, which was further modified by metabolic engineering with metK1-sp and combination of $r m b A$ and $r m b B$ from $S$. peucetius ATCC 27952 to study their effects on spinosyn A and D production.

\section{MATERIALS AND METHODS}

\section{Micro-organisms and vectors}

The bacterial strains and plasmids used in this study are listed in Table 1. The PGEM $^{\circledR}$-T Easy vector (Promega, USA) was used to clone the polymerase chain reaction (PCR) products. pSET152 (Bierman et al., 1992) was used as an integrative vector, and pIBR25 (Sthapit et al., 2004) was used as the expression vector. DNA manipulation was carried out in Escherichia coli XL1-Blue (MRF) (Stratagene, USA), a host cell used for the preparation of recombinants plasmids, was grown in LuriaBertani (LB) broth and maintained on LB agar medium at $37^{\circ} \mathrm{C}$; E. coli JM110 was used to propagate non-methylated DNA. For the selection and maintenance of plasmids, Luria-Bertani (LB) broth and agar plates were supplemented with the appropriate amount of antibiotics (apramycin, $100 \mathrm{mg} \mathrm{ml}^{-1}$ and ampicillin 100 $\mathrm{mg} \mathrm{ml}^{-1}$ ) to grow E. coli. S. spinosa was used for the development of rational strain S. spinosa MUV and S. spinosa MUV was further used for the metabolic engineering.

\section{Culture conditions}

S. spinosa, S. spinosa MUV and recombinants were cultured in different media for different purposes. For regeneration of spore it was cultured on regeneration medium (sucrose $10 \%$, glucose $1 \%$, yeast extract $0.5 \%$, peptone $0.01 \%, \mathrm{MgCl}_{2} \cdot 6 \mathrm{H}_{2} \mathrm{O} 1 \%$, $\mathrm{KH}_{2} \mathrm{PO}_{4} 0.025 \%, \mathrm{CaCl}_{2} \cdot 2 \mathrm{H}_{2} \mathrm{O} 0.3 \%$, agar $2 \%$, pH 6.5 before autoclave and after autoclave $0.2 \%$ of trace element along with $2 \%$ TES buffer was added). The broth cultures were grown at $28^{\circ} \mathrm{C}$ in seed medium (starch $2 \%$, glucose $1 \%$, enzyme hydrolyzed casein $3 \%$, yeast extract $0.3 \%, \mathrm{MgSO}_{4} \cdot 7 \mathrm{H}_{2} \mathrm{O} \quad 0.2 \%$, $\mathrm{KH}_{2} \mathrm{PO}_{4} 0.05 \%$, and $\mathrm{pH} 7.2$ before autoclave). For spinosyn $\mathrm{A}$ and $\mathrm{D}$ production, the media was composed of glucose $4.5 \%$, soybean meal $1 \%$, yeast extract $1.4 \%$, canola oil $2 \%$ and corn steep liquor $0.8 \%$ in distilled water and $\mathrm{pH} 6.8$ was adjusted before autoclave; followed by inoculation with $5 \%$ seed of $S$. spinosa MUV and their recombinants into a baffled 500 -ml flask containing $50 \mathrm{ml}$ of the production medium and grown at $28^{\circ} \mathrm{C}$ on a rotatory shaker at $215 \mathrm{rpm}$ for 8 days.

\section{DNA manipulation and sequence analyses}

PCR premix (Genotech, Korea) or TaKaRa LA Taq ${ }^{\mathrm{TM}}$ (Takara, Japan) was used according to the manufacturer's instructions to amplify the target DNA fragments. PCR was performed in a Thermal Cycler Dice (Takara). The amplification conditions for PCR were: initial denaturation at $94^{\circ} \mathrm{C}$ for $7 \mathrm{~min}, 30$ cycles of denaturation at $94^{\circ} \mathrm{C}$ for $1 \mathrm{~min}$, annealing at $65-70^{\circ} \mathrm{C}$ for $1 \mathrm{~min}$, polymerization at $72^{\circ} \mathrm{C}$ for $1 \mathrm{~min}$; and gap filling at $72^{\circ} \mathrm{C}$ for $7 \mathrm{~min}$. The PCR products were purified and cloned into the PGEM $^{\circledR}-T$ Easy vector for DNA amplification and sequencing. DNA preparation, digestion, ligation and other DNA manipulations were performed using standard techniques for E. coli (Sambrook and Russell, 2001). The chemicals and enzymes used in this study were purchased from Sigma (USA). In silico analyses and comparisons of nucleotide and protein sequences were performed using the BLAST, FASTA, CLUSTALW, and GENEDOC programs. 


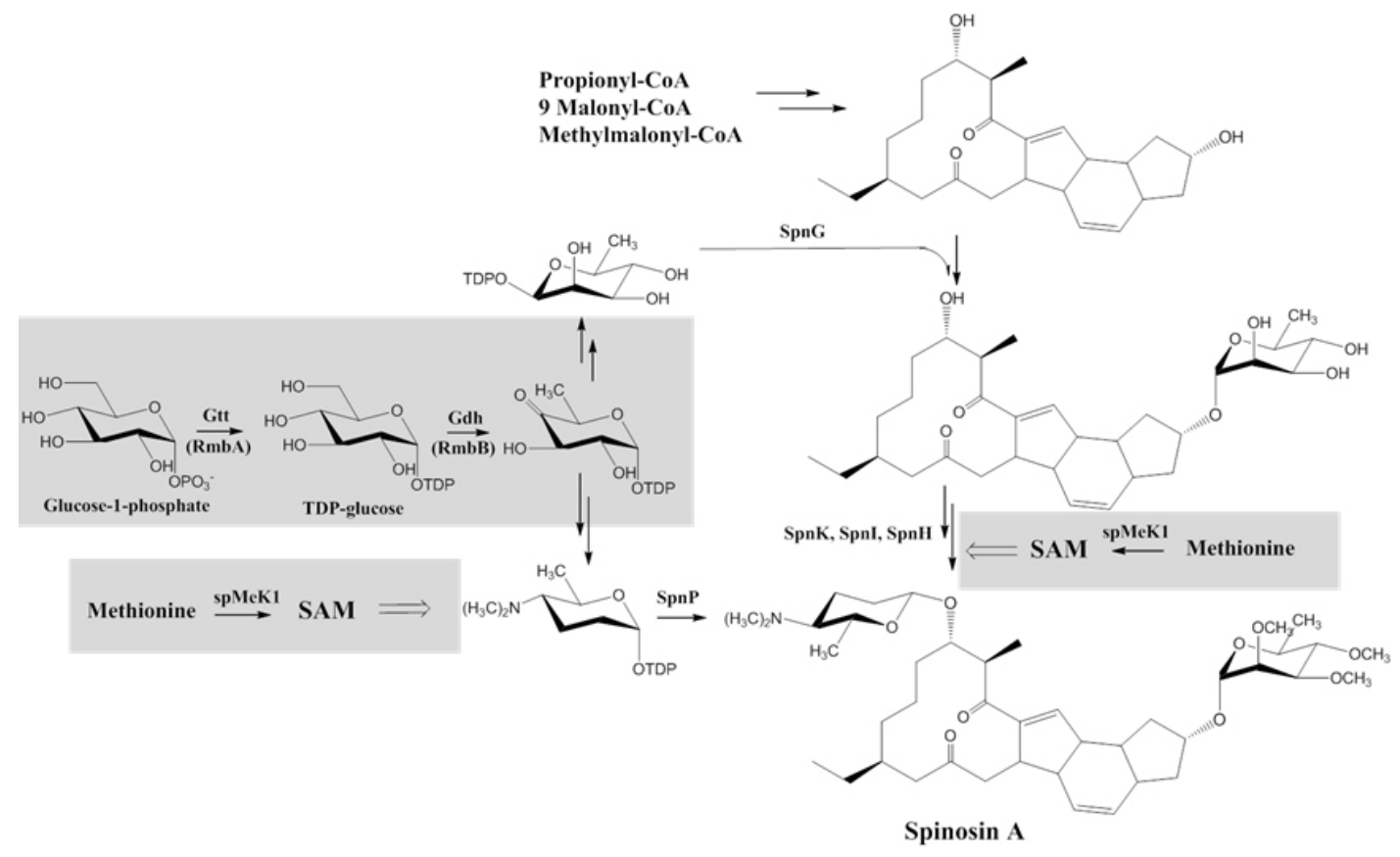

Fig. 1. Schematic diagram of approaches used for enhancement of the spinosyn production in S. spinosa MUV

\section{Construction of the recombinant}

The expression vector, pIBR25 (Sthapit et al., 2004) and integrative vector pSET152, (Bierman et al., 1992) under the control of strong ermE ${ }^{\star}$ promoter, were used for construction of recombinant strains of S. spinosa MUV; using metK1-sp, rmbA, and $r m b B$ for overproduction of spinosad. Oligonucleotides used to amplify metK1-sp (1,209 bp): metK1-sp-F- 5'GGA TCC AAG GAG GAT TCA TGT CCC GTC GTC TCT TCA 3'; metK1-sp-R5'AAG CTT TCA TGC CGC CAC CGC CTT GCG CTT AT 3', rmbA (1,090 bp): rmbA-sp-F- 5'GGA TCC AAG GAG GTC TAC GGT GAA GGC ACT CGT ACT CTC T 3'; rmbA-sp-R- 5'GAA TTC TCA TGA GGA GAT CTG CAC CTT GCT 3', and rmbB (1,010 bp): rmbB-sp-F-5'GAA TTC AAG GAG GTC CAA CAT GAC CAC GAA GAT CCT GGT G 3'; rmbB-sp-R-5'AAG CTT TCA CAG CGC CGC ACG CTC CTT CAG $3^{\prime}$ from genomic DNA of $S$. peucetius for expression vector "pIBR25". Similarly for integrative vector "pSET152", oligonucleotides used to amplify metK1-sp: metK1-sp-F-5'GGA TCC AAG GAG GAT TCA TGT CCC GTC GTC TCT TCA 3'; metK1-sp-R-5'AGA TCT TCA TGC CGC CAC CGC CTT GCG CTT AT 3', rmbA: rmbA-sp-F-5'GAA TTC AAG GAG GTC TAC GGT GAA GGC ACT CGT ACT CTC T 3'; rmbA-sp-R- 5'ACT AGT TCA TGA GGA GAT CTG CAC CTT GCT 3', and rmbB: rmbB-sp-F-5'ACT AGT AAG GAG GTC CAA CAT GAC CAC GAA GAT CCT GGT G 3'; rmbB-sp-R5'GGA TCC TCA CAG CGC CGC ACG CTC CTT CAG 3' from genomic DNA of $S$. peucetius. PCR was performed in a thermocycler (Takara, Japan). The PCR products were purified and cloned into pGEM-T Easy vector and sequenced prior to cloning in the expression vector and integrative vector to verify that no mutation had been introduced during the PCR amplification. After the sequence was analyzed, metK1-sp from $S$. peucetius was cloned into the $\mathrm{BamHI}$ and HindllI sites of pIBR25 and BamHI and Bglll site of pSET152 to form the recombinant plasmid pSIBR and pSAM152, respectively. Similarly, combination of $r m b A$ and $r m b B$ from $S$. peucetius was cloned into the $B a m H I / E c o R I$ and EcoRI/HindllI sites of pIBR25 and EcoRI/Spel and Spel/BamHI site of pSET152 to form the recombinant plasmid pRMBIBR and pRMB152, respectively.

\section{UV mutagenesis}

High producing strain of Saccharopolyspora spinosa was obtained by rational screening procedure of UV mutagenesis. The spores were scraped from the agar surface, using a sterile loop. After filtering through cotton wool, the spore suspension was serially diluted and $5 \times 10^{-5} \mathrm{cfu} \mathrm{ml}^{-1}$ of this suspension was poured and spread onto sterile glass plate containing regeneration medium. The plate without cover was exposed to UV irradiation for $22 \mathrm{~s}$ at the distance of $60 \mathrm{~cm}$ from UV lamp with wavelength of $254 \mathrm{~nm}$ and $40 \mathrm{~W}$. After the UV irradiation, the plates were wrapped with aluminum foil to avoid photoreactivation and incubated at $28^{\circ} \mathrm{C}$ for 8 days. After 8 days of incubation, $99.9 \%$ of the spores were found to be dead, and the remaining colonies were transferred to new plate for stabilization and heavy growth of mutants. Mutants were cultured in seed medium followed by production medium to select the high producing rational strain of Saccharopolyspora spinosa. The high producing rational strain of Saccharopolyspora spinosa was obtained by HPLC analysis and designated as S. spinosa MUV.

\section{Transformation and generation of recombinant strains}

Protoplast transformation was done as previously described (Kieser et al., 2000). The recombinant pIBR25, pSIBR, pRMBIBR, pSAM152 and pRMB152 were propagated in E. coli JM110 to obtain demethylated DNA for transformation in the rational S. spinosa MUV. For protoplast transformation, S. spinosa MUV was cultured in $50 \mathrm{ml}$ of seed medium and $\mathrm{pH} 7.2$ was adjusted before autoclave. After $60 \mathrm{~h}$, the mycelium was harvested by centrifuging (3,200 RPM and $4^{\circ} \mathrm{C}$ for $\left.12 \mathrm{~min}\right)$ and washed with $15 \mathrm{ml}$ of sucrose solution $(10.3 \%)$, then centrifuged (3,200RPM and $4^{\circ} \mathrm{C}$ for $12 \mathrm{~min}$ ), further washed with $15 \mathrm{ml}$ of Pbuffer. Finally, $10 \mathrm{ml}$ of Lysozyme solution $\left(2 \mathrm{mg} \mathrm{ml}^{-1}\right.$ made in $\mathrm{P}$ buffer) was added to cell pellets and incubated the content for $1 \mathrm{~h}$ 

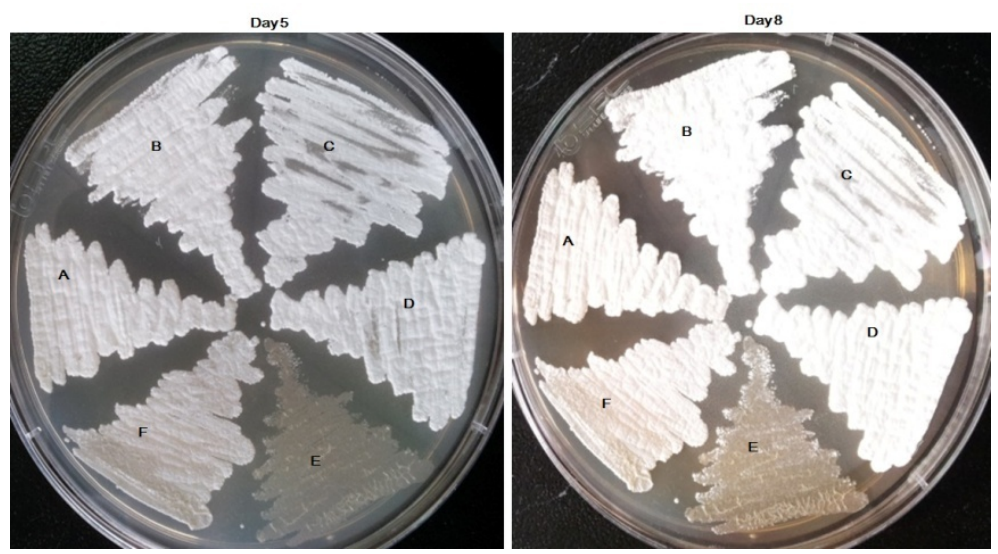

Fig. 2. Morphology of (A) S. spinosa ATCC83543.1, (B) S. spinosa MUV RMB152, (C) S. spinosa MUV RMBIBR, (D) S. spinosa MUV, (E) S. spinosa MUV SAM152, and (F) S. spinosa MUV SIBR on 5 and 8 days.

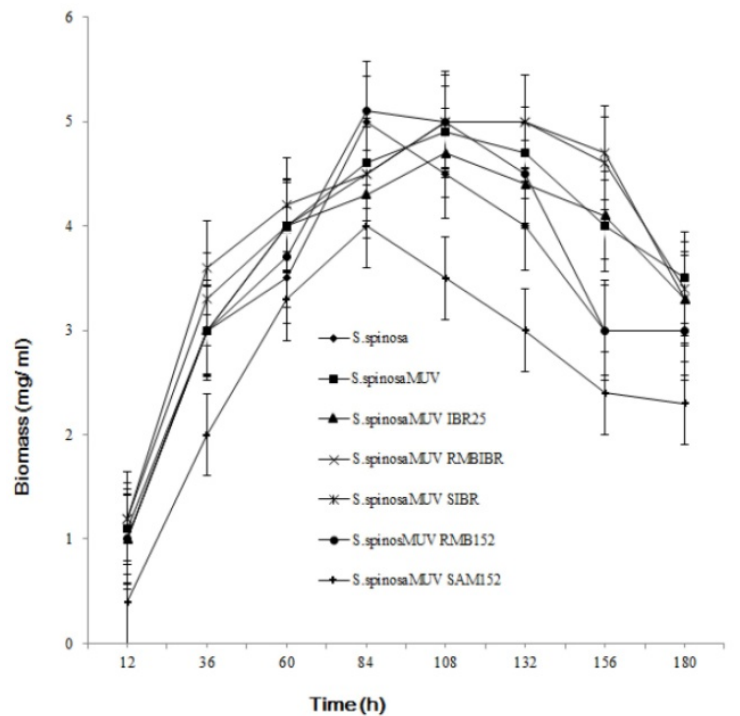

Fig. 3. Time course profiles of biomass obtained by introduction of pIBR25, pSIBR, pRMBIBR, pSAM152, and pRMB152 in S. spinosa MUV along with S.spinosa MUV and S. spinosa ATCC83543.1.

and $45 \mathrm{~min}$ at $37^{\circ} \mathrm{C}$. After incubation, it was filtered and centrifuged for $12 \mathrm{~min}$ at 6,000 rpm, then washed with P-buffer twice, and mixed with $1 \mathrm{ml} \mathrm{P}$-buffer. From the resulting mixture, $100 \mu \mathrm{l}$ was mixed with $20 \mu \mathrm{l}$ of plasmid DNA and $200 \mu \mathrm{l}$ of 40\% PEG1000 and centrifuged for $1 \mathrm{~min}$ to discard the supernatant partially and mixed with $100 \mu$ l of P-buffer, finally it was plated on R2YE plate. The plates were incubated at $28^{\circ} \mathrm{C}$ for $24 \mathrm{~h}$ and then overlaid with $0.3 \%$ agar solution containing10 $\mathrm{g} \mathrm{ml}^{-1}$ thiostreptone and $60 \mu \mathrm{g} \mathrm{ml}^{-1}$ apramycin to select $S$. spinosa MUV recombinant with expression and integrative vector, respectively. After 1 week, thiostreptone and apramycin-resistant colonies were selected and transferred to regeneration medium for further conformation and heavy growth of colonies, then cultured in seed liquid media. Transformation was confirmed by isolation, PCR and restriction enzyme digestion of plasmid from each strain (data not included). The transformants were designated as, S. spinosa MUV IBR25, S. spinosa MUV SIBR, S. spinosa MUV RMBIBR, S. spinosa MUV SAM152, and S. spinosa MUV RMB152 (Table 1). Finally, for the integration of pSAM152 and pRMB152 in chromosomal DNA of S. spinosa
MUV SAM152 and S. spinosa MUV RMB152, respectively, it was cultured up to 4 generation in seed medium with antibiotic. Integration was confirmed by isolation of total DNA of S. spinosa MUV SAM152 and S. spinosa MUV RMB152, followed PCR of metk1sp and $r m b B$.

Analysis of growth on plate and in liquid medium Growth and morphology of all the transformant including $S$. spinosa MUV and wild type was found to similar on regeneration plate media except S. spinosa MUV SAM152 which was found to be growing slower (Fig. 2). To analyze the growth in liquid, fermentation production of Spinosyns was monitored daily, once wild type S. spinosa MUV and all transformants were cultured with $5 \%$ of 36 $\mathrm{h}$ old mycelia harvested from seed medium in different baffled $2 \mathrm{~L}$ flasks containing $0.15 \mathrm{~L}$ of production medium at $28^{\circ} \mathrm{C}$ and 200 rpm for 8 days. After $24 \mathrm{~h}$ of incubation, the cell pellets were collected at $24 \mathrm{~h}$ intervals until $180 \mathrm{~h}$ by centrifuging $10 \mathrm{ml}$ of culture broth at 3,000 rpm for $15 \mathrm{~min}$. The cell pellets were collected, washed with deionized water, and centrifuged twice. They were then placed at $80^{\circ} \mathrm{C}$ until their mass was constant to analyze the growth rate (Fig. 3), which was employed to prove best time for withdrawing spinosyn $A$ and $D$.

Morphological analysis by field emission electron microscope

Wild type, S. spinosa MUV and their transformants was collected after $36 \mathrm{~h}$ from seed medium in different eppendorf tube. The cell pellets were collected by centrifuging $0.5 \mathrm{ml}$ of culture broth at 3,000 rpm for $5 \mathrm{~min}$. The cell pellets were collected, washed with $0.2 \mathrm{M}$ phosphate buffer, $\mathrm{pH} 7.4$, and centrifuged for 2 min thrice. They were prefixed with $2 \%$ glutaraldehyde at room temperature for $1 \mathrm{~h}$ and washed with $0.2 \mathrm{M}$ phosphate buffer, $\mathrm{pH} 7.4$, by centrifuge for 5 times. The prefixed and washed cell was mounted on SAM tube for dehydration and fixation. Dehydration was carried out with increasing concentration of ethyl alcohol $50 \%, 60 \%, 70 \%, 80 \%, 90 \%, 95 \%$ (4 min each) and 100\% (8 min). The dehydrated cells were overlaid with ethanol denaturant (tertbutyl alcohol) at room temperature for $30 \mathrm{~min}$, and were freezedried for $45 \mathrm{~min}$. The resultant freeze-dried sample were coated with platinum at low temperature and examined in high performance field emission electron microscope "JSM-6700F" (CFE SEM) at $\times 5,000$ and $\times 10,000$ (Fig. 4).

Production, extraction and quantification of compound To analyze the production, $5 \%$ seed of wild type, S. spinosa MUV and their transformants were cultured in production media at $28^{\circ} \mathrm{C}$ 
Table 2. Production of spinosyn A and D

\begin{tabular}{lcc}
\hline Bacterial strain & Spinosyn type A $\left(\mathrm{mg} \mathrm{L}^{-1}\right)$ & Spinosyn type $\mathrm{D}\left(\mathrm{mg} \mathrm{L}^{-1}\right)$ \\
\hline S. spinosa ATCC83543.1 & 50 & 27 \\
S. spinosa MUV & 244 & 129 \\
S. spinosa MUV IBR25 & 246 & 129 \\
S. spinosa MUV RMBIBR & 372 & 217 \\
S. spinosa MUV SIBR & 351 & 220 \\
S. spinosa MUV RMB152 & 337 & 217 \\
S. spinosa MUV SAM152 & 230 & 121 \\
\hline
\end{tabular}
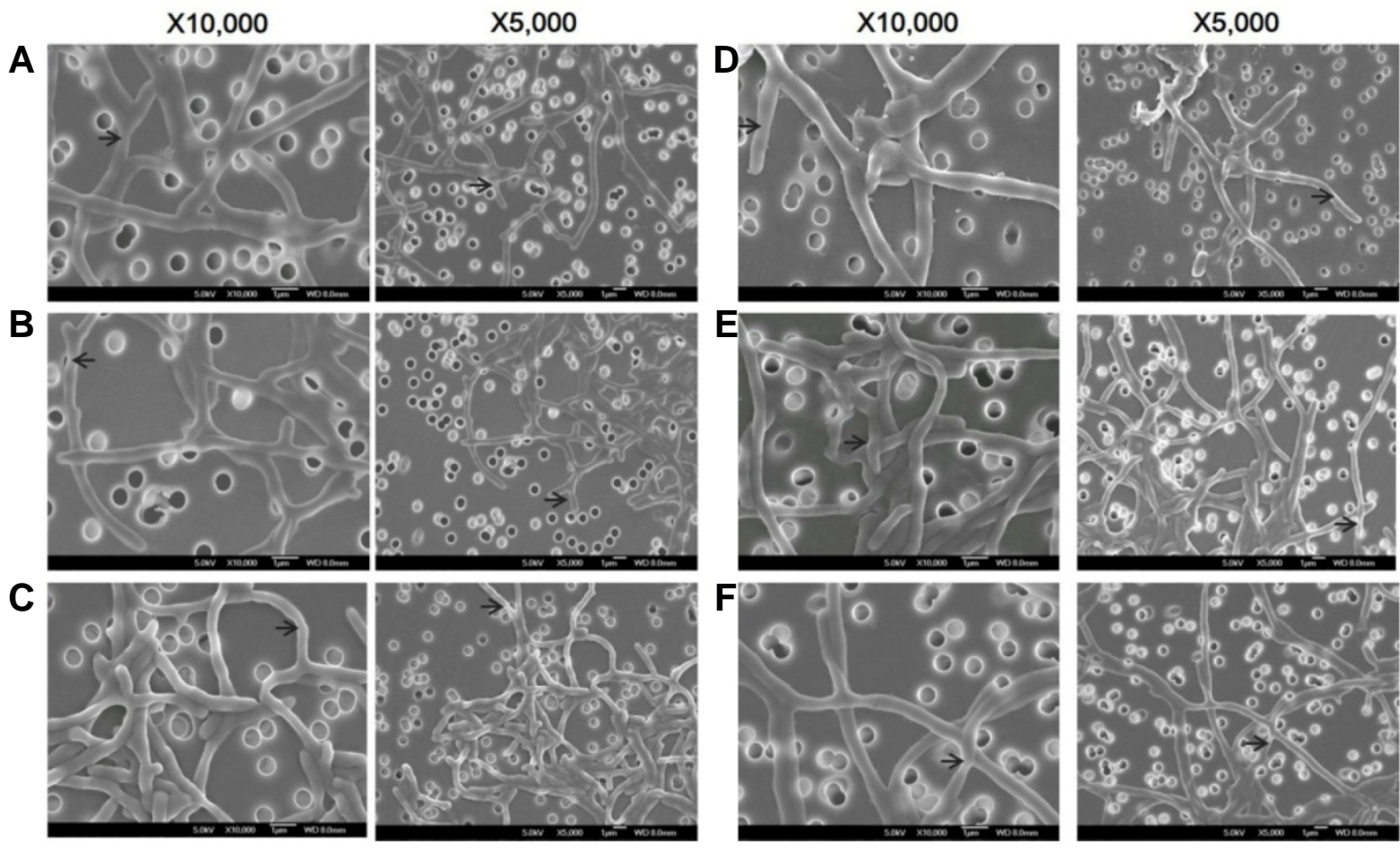

Fig. 4. SEM microscopy of (A) wild type strain, (B) S. spinosa MUV, (C) S. spinosaMUV SIBR, (D) S. spinosa MUV RMBIBR, (E) S. spinosa MUV SAM152, and (F) S. spinosa MUV RMB152. Bars $=1 \mu \mathrm{m}$. The arrows indicate the sites of enhanced spore separation.

for 8 days. One $\mathrm{ml}$ of culture broth of each strain was mixed with 2 $\mathrm{ml}$ of acetonitrile; vortexed for 5-8 seconds and left at room temperature for $30 \mathrm{~min}$ and then centrifuged at $12 \mathrm{~K}$ for $10 \mathrm{~min}$ to remove cell palates. The supernatant was filtrated using $0.22 \mu \mathrm{m}$ syringe filter. The resulting filtrate was injected onto a $\mathrm{C}_{18}$-reversed phase HPLC column $(4.6 \times 250 \mathrm{~mm}, 50 \mu \mathrm{m}$ KANTO Reagents, Japan) under isocratic condition at $245 \mathrm{~nm}$ with flow rate $1 \mathrm{ml} / \mathrm{min}$ and the solvent system used was acetonitrile: methanol: $2 \%$ ammonium acetate buffer $(2: 2: 1, \mathrm{v} / \mathrm{v} / \mathrm{v})$. Spinosyns yields in $S$. spinosa MUV and in their recombinant were determined by HPLC and comparison with a standard spinosyn A and D.

\section{RESULTS AND DISCUSSION}

\section{Construction of recombinant vector}

The $r m b A$ (glucose-1-phosphate thymidylyltransferase), and $r m b B$ (TDP-D-glucose-4,6-dehydratase) from S. peucetius are the enzymes that synthesized TDP-4-keto-6-deoxyglucose as the common intermediate for forosamine and tri-O-methylated rhamnose. Similarly, metK1-sp from S. peucetius plays an im- portant role in the conversion of ATP and L-methionine to Sadenosyl-L-methionine (SAM) and may act as a methyl donor for the transmethylation reaction in the spinosyn gene cluster. Therefore, metK1-sp, and $r m b A$ along with $r m b B$ cloned into pIBR25 and pSET152 under the control of a strong promoter ermE* to construct the recombinant plasmid pSIBR, pSAM152, pRMBIBR and pRMB152, respectively.

\section{Development rational S. spinosa}

Based on earlier studies, UV radiation, in the range of 200-300 $\mathrm{nm}$, produces thymidine dimers and increases probability of deletion during the duplication process; therefore, S. spinosa was exposed to UV at $254 \mathrm{~nm}$ which led to the overproduction of spinosyn $A$ and $D$. The resultant overproducing UV mutant was determined by HPLC analysis and finally, designated as $S$. spinosa MUV.

Construction of recombinant strain of S. spinosa MUV

We developed the recombinant strain of $S$. spinosa MUV by heterologous expression of metK1-sp, and $r m b A$ along with 
rmbB. MetK1-sp was used to make pSAM152 and pSIBR recombinant vector whereas $r m b A$ along with $r m b B$ used to make pRMBIBR and pRMB152. The pSAM152 and pSIBR were transformed into S. spinosa MUV via PEG-mediated protoplast transformation method. The transformed colonies were selected with appropriate concentration of antibiotic (apramycin $60 \mathrm{~g} \mathrm{~m} \mathrm{~m}^{-1}$ and thiostrepton $10 \mathrm{\mu g} \mathrm{m}^{-1}$ ), and plasmid DNA was isolated from selected colonies to conform the transformation. The resultant recombinant of $S$. spinosa MUV was designated as spinosa MUV SAM152 and S. spinosa MUV SIBR, respectively, which plays an important role in the conversion of ATP and Lmethionine to S-adenosyl-L-methionine (SAM) and may act as a methyl donor for the transmethylation reaction in the spinosyn gene cluster that may include the two $\mathrm{N}$-methyl groups of forosamine by spnS, and the three O-methyl groups of tri-Omethylrhamnose by spnK, spnl and spnH (Fig. 1). Similarly, $r m b A$ and $r m b B$ from $S$. peuticus was also amplified and the recombinant plasmid $\mathrm{pRMBIBR}$ and $\mathrm{pRMB} 152$ were constructed under the control of a strong promoter ermE*. Introduction of pRMBIBR and pRMB152 into $S$. spinosa by protoplast transformation generated S. spinosa MUV RMBIBR and S. spinosa MUV RMB152, respectively, which may play the major role to alter the metabolic flux distribution in TDP-4-keto-6-deoxy-Dglucose (Fig. 1), which is the common intermediate for forosamine and tri-O-methylated rhamnose. We also found that chromosomal integration is congenitally stable and remains unchanged through many generations. Indeed, $r m b A$ and $r m b B$ may directly integrate into the promoters of the spinosyns gene cluster; hence strongly activate the genes involved in the spinosad biosynthesis, with an additional stimulatory effect on transcription.

\section{Effect of growth and morphology on production}

Surprisingly, we observed that the morphology of S. spinosa MUV SAM152 was drastically different from that of all the recombinant, S. spinosa MUV, and wild type on regeneration plate (Fig. 2). S. spinosa MUV SAM152 was found to be growing very slowly on plate and in liquid medium which may in turn has insignificant production of spinosyn type $A$ and $D$ in spinosa MUV SAM152, whereas S. spinosa MUV, S. spinosa MUV SIBR, S. spinosa MUV RMBIBR $S$. and spinosa MUV RMB152 exhibited a similar growth curve to the wild type (Fig. 3). Scanning electron microscope (SEM) was used to compare spores of wild-type and mutant strains (Fig. 4). Sample was coated with platinum in place of gold as its molecular mass is smaller than that of gold, which made it easier to study the morphology of cell. We found S. spinosa MUV SIBR, S. spinosa MUV RMBIBR and S. spinosa MUV RMB152 spores were cylindrical like S. spinosa MUV and were smaller in size and larger in number than that of S. spinosa MUV and wild type, which helps to maintain and increase mycelium life sustainability and cell mass, respectively. A good cell mass can be another cause for increased of spinosyn type A and D. Spore observed from spinosaMUV SAM152 was irregular and not distinct which made limited formation vegetative and aerial mycelium, therefore it was found to be slower in growth and lesser in production, whereas wild type spores were round and uniform in size.

\section{Production of spinosyn A and D in recombinant strains}

Recently, it was reported $405 \mathrm{mg} \mathrm{L}^{-1}$ spinosad can be produced where they didn't determine the type of spinosyn (till date) (Xue et al., 2013), but in our investigation we improve the production as well as determine the level of their type. To improve the production of spinosyn A and D, we develop S. spinosa MUV. Production of spinosyn A and D in S. spinosa MUV was $244 \mathrm{mg} \mathrm{L}^{-1}$ and $129 \mathrm{mg} \mathrm{L}^{-1}$, which was 4.88 and 4.77 -fold higher than that of wild-type (Table 2). Further production was carried out in $S$. spinosa MUV via efflux the spinosyn through metK1-sp which in turn found to be producing a higher amount after 6 days in culture and reached in stationary stage at 8 days. The production of spinosyn type $A$ and $D$ was found to be increased by 7.02 and 8.14-fold (351 and $\left.220 \mathrm{mg} \mathrm{L}^{-1}\right)$ in S. spinosa MUV SIBR to that of wild type, respectively, whereas spinosa MUV SAM152 was not significant at 8 days, to that of $S$. spinosa MUV (Table 2). Similarly, $r m b A$ with $r m b B$ play the major role to alter the metabolic flux distribution in TDP-4-keto-6-deoxy-D-glucose (Fig. 1), which is the common intermediate for forosamine and tri-Omethylated rhamnose followed by highest yield of spinosyn $A$ and $\mathrm{D}$ production at 8 days in compared to that of wild type, i.e. increased by $7.44 / 8.03$-fold $\left(372 / 217 \mathrm{mg} \mathrm{L}^{-1}\right)$ and $6.74 / 8.03$-fold (337/217 $\mathrm{mg} \mathrm{L}^{-1}$ ) (Table 2). On the basis of the result, $\mathrm{S}$. spinosa MUV RMBIBR was consider being the highest producing strain which produce $589 \mathrm{mg} \mathrm{L}^{-1}$ of spinosad (spinosyn A $372 \mathrm{mg} \mathrm{L}^{-1}$ and spinosyn D $217 \mathrm{mg} \mathrm{L}^{-1}$ ) (Table 2).

In conclusion; industrially, spinosyn $A$ and $D$ are the most important polyketide. To improve the production of spinosyn A and D, people focused on random mutagenesis (Liang et al., 2009), duplication of gene cluster (Tang et al., 2011), genome shuffling (Jin et al., 2009) and overexpression of clustered genes (Luo et al., 2011; Xue et al., 2013). These techniques have succeeded in generating many industrial strains. Recently, overexpression of clustered genes enhances the production up to $405 \mathrm{mg} \mathrm{L}^{-1}$ spinosad (Xue et al., 2013). Still In terms of the industrial scale, this production is very small and comes with a high production cost. For the same purpose we focuse on strain improvement through random mutagenesis followed by genetic manipulation of the regulatory network. The results showed that application of metabolic engineering on rational strain of $S$. spinosa for the development of recombinant strain; favored as best way to obtain maximum spinosad production $\left(589 \mathrm{mg} \mathrm{L}^{-1}\right)$. On the basis of these results, we conclude that the increased production of spinosad is due to metabolic regulation of genes, which favored as the best way to obtain maximum spinosad production till date and it could be harnessed for the feasible and cost effective production of spinosad at the industrial scale.

\section{ACKNOWLEDGMENTS}

This work was supported by the National Research Foundation of Korea (NRF) grant funded by the Korean government [Ministry of Education and Science Technology (MEST)] (2014R1A2A2A01002875) and supported by a grant from Dongbu Farm Hannong Co., Ltd.

\section{REFERENCES}

Bierman, M., Logan, R., O'Brien, K., Seno, E.T., Rao, R.N., and Schoner, B.E. (1992). Plasmid cloning vectors for the conjugal transfer of DNA from Escherichia coli to Streptomyces spp. Gene 116, 43-49.

Chaudhary, A.K., Dhakal, D., and Sohng, J.K. (2013). An Insight into the "-Omics" based engineering of Streptomycetes for secondary metabolite overproduction. Biomed. Res. Int. 2013, 968518.

Crouse, G.D., and Sparks, T.C. (1998). Naturally derived materials as products and leads for insect control: the spinosyns. Rev. Tox. $2,133-146$.

Huang, K. X., Zahn, J., and Han, L. (2008). SpnH from Saccharopolyspora spinosa encodes a rhamnosyl 4-O-methyltransferase for biosynthesis of the insecticidal macrolide, spinosyn A. J. Ind. Microbiol. Biotechnol. 35 , $1669-1676$.

Jha, A.K., Lamichhane, J., and Sohng, J.K. (2014). Enhancement of herboxidiene production in Streptomyces chromofuscus ATCC 49982. J. Microbiol. Biotechnol. 24, 52-58.

Jin, Z.H., Wu, J.P., Zhang, Y., Cheng, X., Yang, L.R., and Cen, P.L. 
(2006). Improvement of spinosad producing Saccharopolyspora spinosa by rational screening. J. Zhejiang. University SCIENCE A. 7, 366-370.

Jin, Z.H., Xu, B., Lin, S.Z., Jin, Q.C., and Cen, P.L. (2009). Enhanced production of spinosad in Saccharopolyspora spinosa by genome shuffling. Appl. Biochem. Biotechnol. 159, 655-663.

Kieser, T., Mervyn, J.B., Mark, B.J., Keith,C.F., and David, H.A. (2000). Practical Streptomyces genetics (John Innes Foundation Norwich, UK).

Kim, D.J., Huh, J.H., Yang, Y.Y., Kang, C.M., Lee, I.H., Hyun, C.G., Hong, S.K., and Suh, J.W. (2003). Accumulation of S-adenosyl-Lmethionine enhances production of actinorhodin but inhibits sporulation in Streptomyces lividans TK23. J. Bacteriol. 185, 592600.

Kim, H.J., White-Phillip, J.A., Oqasawara, Y., Shin, N., Isiorho, E.A., and Liu, H.W. (2010). The biosynthesis of spinosyn in Saccharopolyspora spinosa: synthesis of permethylated rhamnose and characterization of the functions of SpnH, Spnl, and SpnK. J. Am. Chem. Soc. 132, 2901-2903.

Kirst, H.A., Michel, K.H., Mynderse, J.S., Chio, E.H., Yao, R.C., Nakatsukasa,W.M., Boeck, L.D., Occlowitz, J.L., Paschal, J.W., Deeter, J.B., et al. (1992). Discovery, isolation and structure elucidation of a family of structurally unique, fermentation-derived tetracyclic macrolides. D.R. Baker, J.G. Feynes, and J.J. Steffens, eds. (Synthesis and Chemistry of Agrochemicals III: American Chemical Society, Washington DC, USA), pp. 214-225.

Koffas, M., Roberge, C., Lee, K., and Stephanopoulos, G. (1999) Metabolic engineering. Annu. Rev. Biomed. Eng. 1, 535-557.

Lee, P.C., Umeyama, T., and Horinouchi, S. (2002). afsS is a target of AfsR, a transcriptional factor with ATPase activity that globally controls secondary metabolism in Streptomyces coelicolor A3(2). Mol. Microbiol. 43, 1413-1430

Lee, B.R., Cho, S., Song, Y., Kim, S.C., and Cho, B.K. (2013) Emerging tools for synthetic genome design. Mol. Cells 35, 359370.

Liang, Y., Lu, W., and Wen, J. (2009). Improvement of Saccharopolyspora spinosa and the kinetic analysis for spinosad production. Appl. Biochem. Biotechnol. 152, 440-448.

Luo, Y., Ding, X., Xia, L., Huang, F., Li, W., Huang, S., Tang, Y., and Sun, Y. (2011). Comparative proteomic analysis of saccharopolyspora spinosa SP06081 and PR2 strains reveals the differentially expressed proteins correlated with the increase of spinosad yield. Proteome Sci. 9, 40.

Madduri, K., Waldron, C., and Merlo, D.J. (2001a). Rhamnose biosynthesis pathway supplies precursors for primary and secondary metabolism in Saccharopolyspora spinosa. J. Bacteriol. 183, 5632-5638.

Madduri, K., Waldron,C., Matsushima, P., Roughton, M.C., Crawford, K., Merlo, D.J., and Baltz, R.H. (2001b). Genes for the biosynthe- sis of spinosyns: applications for yield improvement in Saccharopolyspora spinosa. J. Ind. Microbiol. Biotechnol. 27, 399-402.

Nielsen, J. (2001). Metabolic engineering. Appl. Microbiol. Botechnol. $55,263-283$

Okamoto, S., Lezhava, A., Hosaka, T., Okamoto-Hosoya, Y., and Ochi, K. (2003). Enhanced expression of S-adenosylmethionine synthetase causes overproduction of actinorhodin in Streptomyces coelicolor A3 (2). J. Bacteriol. 185, 601-609.

Pan, H.X., Li, J.A., He, N.J., Chen, J.Y., Zhou, Y.M., Shao, L., and Chen, D.J. (2011). Improvement of spinosad production by overexpression of gtt and gdh controlled by promoter $\mathrm{ermE}^{*}$ in Saccharopolyspora spinosa SIPI-A2090. Biotechnol. Lett. 33, 733-739.

Sambrook, J., and Russell, D.W. (2001). Molecular cloning: a laboratory manual, 3rd eds. (Cold Spring Harbor, New York).

Sparks, T.C., Thompson, G.D., Kirst, H.A., Hertlein, M.B., Mynderse, J.S., Turner, J.R., et al. (1998). Methods in biotechnology, biopesticides: use and delivery. F.R. Hall, and J.J. Menn, eds. (Humana Press, USA), pp. 171-188.

Sthapit, B., Oh, T.J., Lamichhane, R., Liou, K., Lee, H.C., Kim, C.G., and Sohng, J.K. (2004). Neocarzinostatin naphthoate synthase: an unique iterative type I PKS from neocarzinostatin producer Streptomyces carzinostaticus. FEBS Lett. 566, 201-206.

Tang, Y., Xia, L., Ding, X., Luo, Y., Huang, F., and Jiang, Y. (2011). Duplication of partial spinosyn biosynthetic gene cluster in Saccharopolyspora spinosa enhances spinosyn production. FEMS Microbiol. Lett. 325, 22-29.

Waldron, C., Madduri, K., Crawford, K., Merlo, D.J., Treadway, P., Broughton, M.C., and Baltz R.H. (2001a). A cluster of genes for the biosynthesis of spinosyns, novel macrolide insect control agents produced by Saccharopolyspora spinosa. Antonie. Van. Leeuwenhoek. 78, 385-390.

Waldron, C., Matsushima, P., Rosteck, P.R.J., Broughton, M.C., Turner, J., Madduri, K., Crawford, K.P., Merlo, D.J., and Baltz, R.H. (2001b). Cloning and analysis of the spinosad biosynthetic gene cluster of Saccharopolyspora spinosa. Chem. Biol. 8, 487499.

Wang, Y., Boghigian, B.A., and Pfeifer, B.A. (2007). Improving heterologous polyketide production in Escherichia coli by overexpression of an S-adenosylmethionine synthetase gene. Appl. Microbiol. Biotechnol. 77, 367-373.

Xue, C., Duan, Y., Zhao, F., and Lu, W. (2013). Stepwise increase of spinosad production in Saccharopolyspora spinosa by metabolic engineering. Biochem. Eng. J. 72, 90-95.

Zhao, X.Q., Jin, Y.Y., and Kwon, H.J. (2006). S-Adenosylmethionine (SAM) regulates antibiotic biosynthesis in Streptomyces spp. in a mode independent of its role as a methyl donor. J. Microbiol. Biotechnol. 16, 927-932. 